\title{
Abstracts Author Index
}

Abbassi, S., A16-02, DBA-06

Abbatiello, B., A06-03

Abdullah, O., A03-04

Abebe, Z., A11-02

Abera, M., B21-02

Abutarboush, R., B02-01

Aceves, A., B09-01

Aceves, M., B04-06, B09-01

Acharya, N., T01-10

Acosta, S., B03-01

Adam, C., T01-15

Adams, S., A01-06

Adegoke, M., A13-01

Adelson, P.D., B08-02

Affeldt, B., A23-03

Afridi, A., B16-05, B16-06

Agimi, Y., B02-10

Ahlawat, A., A05-06

Ahlers, S., A11-23, B02-01, B02-07, B08-21, B08-30

Ahuja, C., T01-01

Aida, V., A22-01

Ajoy, R., A11-04

Akhter, M., B08-10

Akter, F., A03-01

Akula, J.D., A13-11, DBA-05

Ala'i, S., A07-07, B08-07

Alaiyed, S., B18-14

Alan, F., B21-01

Alcazar, O., B06-06

Alcindor, D., A10-05

Algamal, M., A11-04, A20-01, DBA-08

Alger, J., A13-03

Ali, A., B02-12

Ali, S., A11-01

Aljuboori, Z., A22-11

Alkaslasi, M., A11-25

Alshareef, A., T01-02

Amrit, C., B16-06

Amyot, F., A03-07, A13-10, A13-16, A23-07, DBA-01

Andaluz, N., A15-02, B11-02

Andersen, C., A22-03, B06-08

Andersen, C.R., B19-01

Anderson, A., B08-06, DBB-03

Anderson, C., B06-07

Anderson, S., A03-08

Andrews, J., B25-02

Androni, C., T01-07

Angelini, C., A10-02, DBA-03

Ansel, S., A17-01

Anthony, M., A23-06

Antoni, G., T01-21
Anwar, F., B20-03

Aponte, D., B01-05

Aravind, A., B02-05

Arbogast, K., B08-33

Arce, C., A10-05

Argueta, C., B03-01

Armstead, W.M., A22-05

Armstrong, R., A03-02

Arnaud, F., B02-01, B02-02

Arora, T., A07-09

Arroyo, E., A03-03

Arun, P., B02-03

Arvind, A., B02-11

Asarnow, R., A13-03, B25-03

Ashwal, S., A13-02

Assinck, P., T01-18

Atif, F., B17-05

Atkins, C., A18-14, B06-01, B06-06

Atluri, G., B20-03

Auker, C., B17-02

Aussudre, J., B25-01

Avitua, A., A23-03

Awad, A.-W., A16-04

Awwad, H., B08-01

Ayala, C., B18-02

Ayaz, H., A11-11, A23-07

Ayturk, D., A05-06

Büki, A., A22-05

Baban, B., B18-04, B18-05, DBB-06

Babbitt, C., A13-03, B25-03

Babikian, T., A13-03, B25-03

Bachmeier, C., A08-02

Badaut, J., A13-12, B25-01, S08-01

Bader, M., B02-17

Badner, A., A10-02, B27-01, DBA-03

Baer, L., B16-03

Baer, M., A03-10

Baghchechi, M., A23-03

Baig, S., T01-01

Bailey, W., T01-24

Bailey, Z., B02-04

Baine, R., B24-04

Baker, A., A23-01

Balakathiresan, N., A02-01

Balser, D., A06-06

Banks, W., T01-08

Bao, G., B01-11

Bao, Z., A20-04

Bapuraj, J., A11-33

Baratz-Goldstein, R., B26-03

Barbat, S., A11-30
Barmparas, G., A11-25

Barnes, S., A13-02

Barrett, J., B18-03, B21-01

Barretto, T., A23-01

Barriere, G., B25-01

Barsan, W., A16-03

Barse, E., B25-01

Barshikar, S., A11-14

Barton, D., T01-09

Bartsch, A., A07-07

Bashir, A., A11-02

Bass, C., A07-08

Bass, C.R., A22-05

Bassuk, A., T01-20

Batten, S., B27-07

Bauer, A., B29-03

Baxter, G., B29-03

Becker, J.T., A11-08

Beckmann, J., B27-07

Beers, S., A06-10, A11-07, A11-08,

A11-17, A11-18

Beitchman, J., B08-02

Bell, K., A11-14

Bellander, B.-M., B17-03

Bellgowan, P., A07-07

Bellgowan, P.F., B08-07

Benso, S., A06-10, A11-18

Benton, L., B18-14

Benton, M., B02-03

Berdar, N., B01-15

Bergman, T., A06-06

Bergold, P., B06-02

Berke, J., B26-02, DBB-09

Bermudez, S., B21-05

Bernardo-Colon, A., B02-18

Berthiaume, F., A21-04

Bertrand, S., B06-09

Bezin, L., B18-10

Bhaduri, S., B01-12, T01-16

Bhakta, S., A23-03

Bhatnagar, T., A07-02

Bhattarai, D., A23-02

Bholat, O., A01-02

Bhomia, M., A02-01, A06-01

Biekman, B., B08-34

Bigford, G., A22-02

Bilal-Hanif, A., A16-01

Binder, D., A12-06, DBA-04

Birn, R., B08-22

Bishop, E., B23-01

Blaze, J., B02-07

Bleimeister, I., B01-11

Bockhorst, K., A23-02

Numbers refer to abstract numbers, not page numbers. 
Bolding, I., A22-03, A22-09, B06-07, B06-08, B08-12, B08-18, B23-01, B23-04

Bolding, I.J., A18-04

Bondi, C., B01-01, B01-04, B01-08, B01-09

Bondi, C.O., B01-03, B01-07, B01-11

Boone, D., A06-02, A06-17, A22-03, B06-08, B23-01

Borlongan, C., B03-01, B03-02, B27-03

Borrasso, A., A11-07, A11-08, A11-17, A11-18

Boscardin, J., B07-02

Bouix, S., B08-34

Boutte, A., A05-01, A06-03

Bouvard, S., B18-10

Bowman, C., B20-04

Bozdemir, E., B08-31

Braas, D., B20-01

Brabazon, F., B13-01

Bragin, D., A21-01

Bragina, O., A21-01

Brakel, K., B09-01

Bramlett, H., A06-08, A18-01, A22-07, DBA-02, DBA-09

Bramlett, H.M., A22-05

Branca, C., A01-05

Brann, D., B18-08, DBB-07

Braun, M., B18-04, B18-05, DBB-06

Brelsfoard, J., A18-07

Brenner, R., B08-31

Brody, D., B17-01, B29-03

Brody, D.L., B08-08

Broglio, S.P., A11-24

Brooks, J., B27-02

Brooks, W., A01-01

Browning, J., A12-01, A12-03, A18-03, A18-10, DBA-07

Brucks, M., A01-01

Brumley, M., B24-04

Buckley, E., A07-05

Bugay, V., B08-31

Bullock, K., T01-08

Bullock, R., A05-03, A05-10

Bulock, R., T01-07

Bunge, M.B., A02-02, B30-03

Burke, J., A11-36

Burke, J.F., B01-10

Burnett, B., B21-02

Burns, M., B08-28, B18-03, B18-14, T01-09

Burri, D., A06-06

Butman, J., A09-01, A13-13, A13-14

Butoryak, M., A11-18

Buttles, S., B16-07

Button, E., A11-03

Byrnes, K., B01-02, B21-05

Byro, M., A22-06, B20-02, S01-01

Cabrera, I.B., B18-01

Cai, Y., A06-12, A11-05, A13-15, B01-12, T01-16

Calixte, D., B06-06

Camarillo, D., A11-30, B08-36

Camarillo, D.B., A07-07

Campbell, R., B08-04, B08-33
Canac, N., A11-27

Cao, Y., A12-01, A12-03

Caprelli, M., A06-04

Cardenas, A., B02-08

Cardenas, J., B16-03

Cardiff, K., A05-04, B16-07

Carlson, A., B11-02

Carlson, S., B29-01, B29-02

Carr, W., B02-07

Cartagena, C., A05-01

Casa, D., B08-34

Castillo, G., B13-02

Castillo, M., B22-02

Catheline, G., B25-01

Caudle, K., A12-01, A18-03, A18-10, DBA-07

Cavanaugh, J., B02-19

Cavazos, J., B08-31

Cerqueira, S.R., A02-02, B30-03

Chakraborty, D., A11-10

Chamankhah, M., A10-02, DBA-03

Chan, L., A13-13, B08-29

Chan, P., A10-02, DBA-03, T01-01

Chancey, C., A11-20

Chandra, N., B02-05, B02-11, B02-12, B02-13, B02-16

Chang, J., A18-12, B22-01

Chang, Y., A06-10, A11-17

Chao, H., A20-04

Chapman, J., B02-15

Chapple, K., T01-03

Charles, A., A16-01

Charlton, J., B08-03

Chaturvedi, S., A12-06, DBA-04

Chavda, N., A20-02

Chavko, M., B02-09

Chen-Roetling, J., B16-01

Chen, J., A15-01

Chen, P., A15-01

Chen, S., B28-02

Chen, Y., B02-01, B02-02

Cheng, C., B24-05

Cheng, J., B01-03, B01-08, B01-09, B07-02

Cheng, J.P., B01-07

Cheng, W.H., A11-02, A11-03

Cherian, L., A06-17

Cherukuri, K., B07-01

Chervu, N., B08-32

Cheung, L., B27-06

Chi, N.-C., B24-05

Chiluwal, A., A01-02, B16-05

Cho, N., A11-25

Choe, M., B08-23, DBB-05

Choi, A., A22-11

Choi, B., B02-07

Choi, H.A., A22-04

Choi, T., A01-06

Chong, A., A06-14

Chou, Y.-Y., A13-10, A13-13, A13-16

Chou, Y.-y., A03-07, DBA-01

Chrastek, M., A06-06

Christian, G., A16-06

Christian, S., A01-01, A18-05
Christoforou, C., B02-03

Chung, J., B04-07

Ciarlone, S., A11-23

Citron, B., A18-12, B22-01

Claridge, J., A10-04

Clark, A., B02-18

Cleveland, R., B08-06, DBB-03

Clifton, J., B02-18

Cloud, A., B20-06, B27-07

Cnossen, M., A11-34, A11-35

Coats, B., A03-04

Coats, J., A13-12

Cochran, S., B03-01

Cognetti, J., T01-10

Cohen, A., B11-01

Cohen, A.S., B01-10

Cohen, H., B02-20, B02-21

Coksaygan, T., A16-10

Col, N., A16-05

Coll-Miro, M., A18-16, B24-02

Collins, M., A11-07, A11-08, A11-17, A11-18

Conley, Y., B15-01

Conti, A., B08-03

Converse, M., A23-04

Cook, A., T01-03

Cook, D., T01-08

Coolen, L., B26-06

Cornelison, R.C., B30-03

Costine, B., B25-02

Cota, M., A09-01, B08-13, B08-17, B08-29

Cotton, B., B16-03

Covert, H.W., A03-05

Cox, C., B25-06

Cox, D., A18-11, B26-01

Cox, M., B25-05

Crane, P., S04-01

Crawford, E., B25-02

Crawford, F., A08-02, A11-04, A20-01, B01-05, B08-24, DBA-08

Cripton, P., A06-16, A11-02, A11-03

Cromer, J., B08-04

Crynen, G., A08-02, A11-04

Cullen, D.K., B03-03, B11-04, DBB-01

Cullen, K., T01-10

Curtin, J., A04-02, B30-02

Dai, H., A18-15

Dai, Y., A11-14, B30-01, B30-02

Dalgard, C., B21-02

Dalton, J., T01-03

Daly, S., A06-06

Dambinova, S., PL01-04

Dammavalam, V., A06-06

Daniel, K., B18-11

Darowish, M., A04-03

Darrow, D., A09-02, A14-01

Dash, P., A21-06, A22-04, A22-05

Datwyler, S., A06-06

Dauser, R.C., A02-02

Davenport, R., B29-03

David, B., B30-01, B30-02

Davies, M., B17-01 
Davis, J., B16-02, B24-04

Davis, R., A08-01

Davis, T., B08-17

Dayaram, K., A22-12

Debert, C., B08-27

Deborah, S., B16-07, B23-02

DeDominicis, K., A05-01, A18-03

DeFazio, R., A23-05

Deibert, C., B27-02

Deiter, G., A19-01

De la Tremblaye, P., B01-04

de la Tremblaye, P.B., B01-03, B01-07, B01-09, B01-11

Deng-Bryant, Y., A05-07, B23-02

Deng, H., A05-11, A11-34, A11-35, A11-36

Dennis, E., A13-03, B25-03

DePasquale, E., B20-03

de Rivero Vaccari, J.P., A16-02, DBA-06, T01-07

DeSana, A., B23-03

Desai, N., B27-06

DeVeau, K.M., B13-01

DeWitt, D., A06-02, A06-17, A22-03, A22-09, B06-07, B06-08, B08-18

DeWitt, D.S., A11-01, A18-19, A22-05, B02-14, B19-01

Dewey, E., B16-04

Dewitt, D., B19-02, B23-01

Dhall, S., A05-11

Dhandapani, K., B09-02, B18-04, B18-05, B18-08, DBB-06, DBB-07

Dhillon, N., A11-25

Di, L., B18-13

DiNapoli, V., B11-02

Diamond, D., A20-01, DBA-08

Diaz-Arrastia, R., A03-07, A13-10, A13-16, A23-07, DBA-01

Diaz, R., A11-27

Dieppa, M., A11-14

Dietrich, D., A16-02, A22-02, DBA-06, T01-07

Dietrich, W.D., A06-08, A18-01, A18-14, A22-05, A22-07, B06-06, DBA-02, DBA-09

Dikmen, S., B01-13

Ding, K., A11-14

Ding, Q., T01-06, AANS02-02

Dixon, C.E., A06-08, A18-02, A22-05, A22-07, B29-01, B29-02, DBA-02, DBA-09

Dixon, E., WS06-03

Dodd, A., B08-04, B08-33

Dolle, J.-P., A03-05, A03-08

Donovan, A., A22-02

Doran, S., B21-01

Dougherty, J., A05-09

Douvdevani, A., B02-21

Dragas, R., B28-01

Dreier, J., B11-02

Driscoll, D., A18-12

Driwech, W., B02-03

Druzgal, J., B08-05, B08-26

Duhaime, A.-C., B25-02
Duma, S., A07-03, A11-21

Dunn, T.J., A11-01

Eaton, J., A16-01

Ebadi, H., A05-02

Edelman, K., A06-10, A11-07, A11-08, A11-17, A11-18

Edmiston III, W., B04-07

Edwards, A., B08-01

Ehinger, J., A22-06

Eiferman, D., B18-07

Eken, O., B02-03

El Demerdash, N., B25-04

Eldahan, K., A18-11, B26-01

Elder, G., B02-07

Eliamani, S., A23-03

Elliott, M., T01-10

Ellis, M., B25-03

Elmér, E., A22-06

Elpaz, A., B26-03

Emery, C., B08-27

Emhoff, T., A05-06

Empey, P., A18-02, A22-07, DBA-09

Enjalric, I., A23-03

Erickson, B., A11-20

Esenaliev, R., A16-09, A22-09, B08-12, B23-04

Esenaliev, R.O., A18-04

España, L., B08-22

Esparza, T.J., B08-08

Espindola, D., A07-04

Espinosa-Garcia, C., B17-05

Espinoza, L., B08-31

Espinoza, T., A11-10, B08-35

Esposito, E., S01-03

Essepreis, J., A21-02

Esterlitz, J., A07-07, B08-07

Evans, D., A13-04

Evans, K., A11-10, B08-35

Evanson, N., B20-03

Eynde, J.O.'t., A07-08

Faden, A., A18-16, B04-03, B04-04, B04-05, B18-03, B24-02, B27-06, DBB-02

Fahlström, M., T01-21

Fair, K., B16-04

Fanselow, M., A20-02

Fanton, M., A11-30

Farzaneh, N., A11-32, A11-33, B07-05

Fatima, S., B09-02

Fehlings, M., A10-02, B27-01, B28-01, DBA-03, T01-01, T01-22

Feldman, K., A18-02

Fels, S., A07-02

Feng, K., B02-19

Ferguson, A., A11-36, B07-02, T01-06, AANS02-02

Ferguson, A.R., A22-05, B19-01

Ferguson, P., T01-20

Ferguson, R.D., A13-11, DBA-05

Ferguson, S., A08-02, B01-05, B08-24

Fievisohn, E., B02-06

Fijal, P., A07-01

Finan, J., A03-06
Fine, D., B01-06

Finley, S., A21-03

Fisher, A., A11-28, B08-06, DBB-03

Fisher, A.M., A13-11, DBA-05

Fiskum, G., A16-10

Flerlage, W., A05-09

Flesher, K., A03-07, A13-16, DBA-01

Flores, A., A05-09

Floros, T., B08-08

Floyd, C., A22-01, B08-25, B23-05, B24-01, B24-03, T01-11, WS06-03

Floyd, R., B08-32

Folkerth, R., B05-01

Folkerth, R.D., A05-05

Folkerts, M., T01-14

Folweiler, K., B11-01

Foreman, B., A15-02, B11-02

Forman, J., T01-02

Fountain, E.M., B08-08

Frank, J., B20-05

Franz, E., A11-28, B08-06, DBB-03

Franz, E.S., A13-11, DBA-05

Free, K.E., B01-03

Freund, J., B06-01

Frey, W., B01-02

Friedman, A., A06-14, A11-28

Friess, S., B17-01

Fu, A., B01-14

Fujiyama, Y., A06-13

Fukuda, A., A13-12

Fullerton, J., A17-01

Fulp, J., A18-13

Furlow, W., B18-14

Furones-Alonso, O., A18-01

Furuya, T., A10-01, A10-03, A13-08, A21-09

Gabler, L., A07-03

Gajavelli, S., A05-03, A05-10, T01-07

Gajewski, B., A16-03

Galicich, W., A06-06

Gallagher, D., A23-01

Galuta, A., B28-02

Gancayco, C., B08-05

Ganetzky, B., S02-03

Gangolli, M., B08-08

Gao, J., A11-01

Gardner, R., A01-03, A01-07, B07-02

Gaski, G., S02-02

Gaudette, E., B08-09

Gaupp, E., A11-15

Gay, K., A07-07

Gayen, M., A02-01, A06-01

Ge, Y., B02-07

Geddes, J.W., A22-12

Gee, J., A16-04

Gensel, J., T01-24

George, A., B22-03

George, K., B18-14

Georges, B., B18-10

Ghazarian, N., A16-06

Ghebremedhin, M., B02-08

Ghinda, D., B28-02

Ghosh, N., A13-02

Gilliam, W., T01-25 
Gilsdorf, J., A06-03, A18-03, A22-07, B23-02, DBA-09

Gimlin, K., B06-05

Giordano, K., B08-10

Gist, I., B02-03

Giudice, J.S., T01-02

Giza, C., A11-06, A13-03, A20-02, B08-23, B25-03, DBB-05, T01-14

Giza, C.C., A06-12, A13-15, B01-12, T01-16

Glaser, T., A20-03

Glenn, T., B20-01

Glowatzki, E., A04-02

Glueck, A., B08-30

Glushakov, A., B03-02, B27-03

Glushakova, O., B03-02, B27-03

Godbout, J., B18-07

Godzik, J., T01-03

Goldberg, D., B30-02

Goldberg, R., A16-05

Goldman, D.P., B08-09

Goldstein, L., A11-28, B08-06, DBB-03

Goldstein, L.E., A13-11, DBA-05

Gollihue, J., A18-11

Golub, V., B24-04

Gong, H., B22-03

Gonzalez, S., A11-31

Goodkin, H., B08-26

Goodrich, J., A11-23, B08-30

Gopinath, S., A14-02, A16-07

Gore, R., A11-10, B08-35

Gorse, K., A11-09, B08-20

Goslin, B., B08-11

Grady, M., A11-11, A11-12, B08-33

Grant, A., B08-12

Grant, G., B08-36

Grant, S., A06-03

Grau, J., B16-02, B24-04, T01-13

Greco, T., B20-01, T01-14

Green, R.S., A12-04

Greenberg, S., B02-06

Greene, A.M., B01-03

Greig, N., A18-12, B02-17

Griesbach, G., A21-03, B13-02

Griffin, A., B08-13

Griffiths, D., A21-05, B08-02, B18-06

Grill, R., B28-04

Grovola, M., B11-04

Grudziak, J., A16-01

Grusky, A., A11-06

Gu, M., B02-09

Gullapalli, R., B08-16

Guptarak, J., A18-04, B08-12

Guptarat, J., A18-19

Gupte, R., A01-01, A18-05

Gurganus, J., A05-02

Gurkoff, G., B06-04, B06-05

Gurney, M., A18-14, B06-06

Guzowski, N., T01-04

Haacke, E.M., B08-16

Haar, C.V., B01-15

Haber, M., A03-07, A13-10, A13-16, DBA-01

Hacker, J., B27-01

Haddad, E., A23-03
Haghighi, F., B02-07

Haines, S., A14-01

Hajiaghamemar, M., A06-05

Halford, J., A06-14, A06-16

Hall, A.B., B08-03

Hall, E., A18-06, A18-07, A18-09, WS06-03

Hall, E.D., A22-05

Hamer, M., A23-03, A23-05

Hamilton, A.G., B13-01

Hamilton, R., A11-27

Hammoud, D., B20-05

Han, I., A22-11

Handa, R., B12-01

Hanscom, M., B27-06

Hansel, G., A03-08

Hansson, M., A22-06

Haque, A., B17-02

Harbaugh, K., A04-03

Haro, P., A11-25

Harris, J., A01-01, A18-05

Harris, N.G., A06-12, A13-15, A21-08, B01-12, T01-12, T01-16

Hartings, J., A15-02, B11-02

Haung, Y.-J., B 16-02

Hausser, N., A22-09

Hawkins, B., A22-03, A22-05, B06-07, B06-08, B08-18, B19-02

Hawkins, B.E., B19-01

Hawryluk, G., A13-04, A16-04, AANS02-02, T01-06

Hayakawa, K., S01-03

Hayes, R., A06-08, A22-07, B03-02, B08-20, B27-03, DBA-02, DBA-09

Hayes, R.L., A22-05

Hazel, T., A05-03, A05-10

Hazzard, B., B17-02

He, J., A 10-04

He, L., B18-13

Hefti, M., B05-01

Hegdekar, N., B04-05

Heinrichs, S., A20-03

Heiskanen, M., A06-09

Helkowski, M., B01-11

Hellal, F., B06-03

Hellmich, H., A06-02, A06-17, A22-03

Hemphill, M., T01-05

Henchir, J., B29-01, B29-02

Hendershot, K., A11-10, B08-35

Henley, K., B24-01

Henry, R., B18-03, B21-01

Hergenroeder, G., A22-04

Hernandez, P., B06-05

Herrera, J., A23-02

Hess, D., B18-04, DBB-06

Hiba, B., B25-01

Hill, C., B30-01, B30-02

Hill, R., A18-06, A18-07

Hinds, C.S.R., A22-05

Hinson, H., A13-05, B16-04

Hinzman, J., B11-02

Hirschi, R., T01-06, AANS02-02

Hlavac, N., A02-03

Ho, L.T.V.T., B17-02

Hoane, M., B27-08
Hobbs, M., B08-31

Hockenbury, N., B01-02

Hoda, M.N., B09-02, B18-04, B18-05, DBB-06

Hoffer, B., B02-17

Hoffman, A., A20-02, B08-02

Holcomb, J., A01-06, B16-03

Holden, R., A21-02, A21-06, A21-07

Holloway, A., B09-03

Hollowell, L., B08-34

Holmes, G., A19-01, B17-04

Holshouser, B., A13-02

Holstein, D.M., B08-31

Hong, J., A10-02, B27-01, B28-01, DBA-03

Hong, S., B09-03

Hong, T., A18-18

Hook, G., A06-03

Hook, M., B04-06, B09-01

Hook, V., A06-03

Horiuchi, M., B26-02, DBB-09

Horn, A., B08-32

Horsmon, M., A05-02

Horvath, T., B04-01

Hovda, D., A20-02

Howell, S., A21-03

Hsieh, E., A20-02

Hu, Z., A05-03, A05-10

Hua, N., A11-28

Huang, H.M., B16-07

Huang, Y.-J., B24-04, T01-13

Hubbard, B., B08-14

Hubbard, M., A06-06

Hubbard, W., B20-06

Huber, B., A11-28

Huber, B.R., A13-11, DBA-05

Huber, D., B08-15, DBB-04

Hudson, M., A21-02

Hugerth, M., A22-06

Huie, J.R., AANS02-02, B19-01, T01-06

Hulbert, L., WS06-03

Hur, Y., B08-02

Hwang, H., A05-01, A05-07

Hylin, M., A21-02, A21-06, A21-07, B21-04, B25-05

Ibrahim, W., B20-05

Ichkova, A., B25-01

Iijima, Y., A10-01, A13-08

Iivarinen, J., A06-07

Ijima, Y., A10-03, A21-09

Ikonomovic, M., A06-10

Inage, K., A10-01, A10-03, A13-08, A21-09

Ipapo, K., B08-09

Iqbal, H., A22-12

Iraji, A., B08-16

Irimia, A., A13-06, A13-07

Iring, S., B02-05, B02-13

Ivins, B., B02-10

Izadi, A., B06-05

Izzy, S., A05-06

Jackson, M., B25-06

Jackson, T., A03-09

Jacobs, A., B17-01 
Jacobs, E., B27-08

Jacobs, K., A03-10

Jacobsen, S., A06-03

Jaiswal, S., B01-02

Jeronimo, E., B02-09

Ji, J., A20-04

Ji, S., A11-05, B08-36

Ji, X., S01-03

Jiang, C., A11-33

Jikaria, N., B08-13, B08-17, B20-05

Jin, $X .$, T01-23

Jin, Y., A13-03, B25-03

Johansson, P., B16-03

Johnson, B., B01-10

Johnson, C., B08-23, DBB-05

Johnson, D., B08-32

Johnson, J., A13-03, B25-03

Johnson, K., A22-03, A22-09, B06-07, B06-08, B08-12, B08-18, B23-01, B23-04

Johnson, K.M., A18-04

Johnson, T., A11-18

Johnson, V., A03-03, B03-03, B11-04, DBB-01, T01-10, T01-15

Johnson, V.E., A17-01

Jones, G., B18-11

Jones, J., B04-03, B04-05, DBB-02

Joseph, B., B08-14

Joseph, J.R., A11-24

Joseph, K., A07-07, B08-07

Jullienne, A., A23-03

Juras, A., B08-14

Kaczmarek, L., B04-01

Kakulavarapu, R., B02-11

Kakulavarapu, R.R., B02-13

Kallakuri, S., B02-19

Kamimori, G., B02-08

Kamins, J., A11-06

Kane, M., B04-03, B04-05, DBB-02

Kang, H., A05-09

Kannan, S., B06-09

Kaplan, G., A20-03

Kaplan, Z., B02-20, B02-21

Karlsson, M., A22-06, B20-02

Karttunen, J., A06-09

Kashyap, T., B03-01

Katzenberger, R., S02-03

Kaup, A., A01-07

Kawoos, U., B02-01, B02-09

Keane, R., A16-02, DBA-06, T01-07

Keeley, K., B22-01

Kelestemur, H.F., PL01-02

Kelley, A., A11-20

Kelly, M., A10-04

Kemppainen, S., A06-07, B10-01

Kenney, K., A03-07, A13-10, A13-16, DBA-01

Kerr, N., A16-02, DBA-06

Keselman, P., A01-01, A18-05

Key, R., B25-05

Khan, M.B., B18-04, DBB-06

Khan, M.W., A16-05

Khan, Z., B18-04, DBB-06

Khazaei, M., T01-01
Kibayashi, K., B04-02

Kilbaugh, T., A06-05, A22-06, B20-02, S01-01

Kim, C., B28-03

Kim, D.S., B02-17

Kim, J., A18-08, B28-03

Kim, J.-H., B29-03

Kim, S., B29-03

Kim, Y., A18-08

Kimberly, B., A01-08

King, A., B02-19

Kinney-Lang, E., A23-05

Kinsler, R., A06-16

Kitagawa, R., A22-04

Kitamura, M., A10-01, A10-03, A13-08, A21-09

Kiyohira, M., A06-13

Kleeberger, A., A06-06

Kline, A., B01-01, B01-04, B01-07, B01-08, B01-09

Kline, A.E., A21-08, B01-03, B01-11

Klotz, A., B08-19

Knollmann-Ritschel, B., A02-01, A06-01

Koch, P., B11-04, T01-15

Kochanek, P., A03-09, A22-07, B08-20, DBA-09

Kochanek, P.M., A06-08, A18-02, A22-05, DBA-02

Koda, M., A10-01, A10-03, A13-08, A21-09

Koehler, R., B06-09, B25-04

Koh, E., B04-03, B04-04, DBB-02

Kokiko-Cochran, O., B18-07

Koliatsos, V., A04-02, A05-02, A11-31, B22-02, T01-25

Kontos, A., A11-07, A11-08, A11-17, A11-18

Korley, F., A06-14, A11-33, A16-03

Korol, T., B27-03

Kosoris, N., B08-35

Kota, D., B28-04, B28-06

Kotermanski, S., A03-09

Kou, Z., B08-16

Koutsouras, G., A01-02

Kreber, L., B13-02

Krieger, D., A11-07, A11-08

Kroner-Milsch, A., B27-04

Kudchadkar, S., B06-09

Kulbe, J., A18-06, A18-09

Kumar, A., B18-03, B24-02

Kumar, R., A06-11, A06-15, A12-02, B07-03, B07-04, T01-17

Kumar, S., A21-04

Kummer, T., B29-03

Kundu, B., A16-04

Kuo, C., B08-36

Kurt, M., A11-30

Kutash, L., B01-01, B01-08, B01-09

Kuwar, R., B18-13

Kwiatkowski, A., B02-06

Kwon, B., A06-16

LaPlaca, M., A11-10, A11-15, B08-35, WS06-03

LaPlaca, M.C., A22-05

LaPorte, M., B01-01, B01-11
LaValle, C., B02-08

Labastida, J.A., A11-01

Lafrenaye, A., A06-08, A11-09, A22-07, B08-20, DBA-02, DBA-09

Lai, Y., B27-05

Lajud, N., B01-03, B01-07, B01-09

Laksari, K., A11-30

Lam, J., A20-02

Lamb, B., A11-28

Lantzy, M.K., A11-09

Lapointe, A.P., A11-24

Lara, D., A21-01

Larsson, E.-M., T01-21

Lashoff-Sullivan, M., B02-01

Latour, L., A09-01, B08-13, B08-17, B08-29

Law, L., B21-03

Law, L.M., A21-05

Lawrence, C., B08-17

LeDoux, D., A01-02

Leal, G., B24-04, T01-13

Leary, P., A08-02, A11-04

Lechleiter, J., B08-31

Leddy, J., B08-33

Lee, D., A03-10

Lee, E., B28-03

Lee, H., B28-03

Lee, K., A18-08

Lee, L., A11-10, B08-35

Lee, M., B03-01

Lee, S., A05-10, B28-03, T01-07

Lee, S.T., B28-03

Lee, Y.-S., B30-03

Leite-Morris, K., A20-03

Lerner, M., B08-01

Leung, L.Y., A05-04, A05-07, A12-01, B16-07

Levin, H., A06-02, A11-13, B08-34

Lewis, M., A13-09

Lewis, T., A11-14

Ley, E., A11-25

Li, C., B16-05, B16-06

Li, H., B02-16

Li, J., A03-08, B07-01

Li, L., A18-18

Li, S., B26-02, DBB-09

Li, Y., B02-17, B04-03, B04-04, B24-02, B29-02, DBB-02

Li, Z., A20-04, B02-18

Li, Z.Z., A06-01

Liao, Z., A12-01, A12-03

Lichtman, A., B18-12, DBB-08

Lifshitz, J., A01-04, A01-05, A21-05, A22-10, B08-02, B08-10, B18-06, B21-03

Lin, C., A20-04

Lin, T.-H., B22-03

Lingsma, H., A11-34, A11-35

Lipinski, M., B04-03, B04-04, B04-05, DBB-02

Littlejohn, E., B23-03

Liu, B., A11-10, B08-35

Liu, E., A23-01

Liu, J., A07-01, A07-06, A12-06, B11-03, DBA-04, T01-18

Liu, N., A20-04 
Liu, N.-K., B27-05

Liu, S., B04-03, B04-04, B04-05, DBB-02

Liu, T., B02-16

Liu, Y., A20-04, S01-03

Lizhnyak, P., A22-08, DBA-10

Lo, E., S01-03

LoPrestgi, M., B02-07

Loane, D., B18-03, B21-01, B27-06

Lockman, P., A05-08

Lockyer, J., A11-12

Logsdon, A., A05-08, T01-08

Long, J., B02-03

Long, J.B., A22-05

Long, M., B02-11

Loo, J., A06-16

Lopez, E., B16-03

Lu, X.-C., A12-01, A12-03

Lu, X.-C.M., A18-03

Lucke-Wold, B., A05-08

Lukas, R., B18-06

Lule, S., B04-07

Lungmus, C., A20-01, DBA-08

Lynch, C., A08-02

Ma, E., B27-06

Ma, L., B26-02, DBB-09

Ma, M., B18-08, DBB-07

Ma, X., A18-02, B29-02

Machamer, J., B01-13

Madathil, S.K., A18-03, B23-02

Magnuson, D.S.K., B13-01

Mahan, M., A06-06

Mahavadi, A., A05-03

Mahoney, J., T01-20

Main, B., B08-28, T01-09

Major, S., B11-02

Maki, S., A13-08

Malik, S., B02-10

Mallela, A., T01-10

Malone, M., B08-36

Manley, G., A01-03, A01-07, A05-11, A11-16, A11-34, A11-35, A11-36, AANS02-02, B01-13, B07-02, B08-09, T01-06

Mannix, R., B08-33

Mao, X., B06-03

Margulies, S., A06-05, A22-06, B20-02, S01-01

Mari, N.S., A13-15

Maria, N.S., A06-12

Marino, J., A06-06

Marion, C., A03-02

Marion, D., B02-10

Marklund, N., T01-21

Marshall, I., B01-01, B01-08, B01-09

Martens, K., A11-02, A11-03, B01-15

Martin, R., A16-03

Martinez, A.T., B08-17, B08-29

Martinez, L., A11-27

Master, C., A11-11, A11-12, B08-33

Master, S., A11-12

Matar, M.A., B02-20, B02-21

Matthews, C., A11-20

Matthiesen, L., B18-01

Mattucci, S., A07-01, A07-06, B11-03
Matyas, J., A18-16, B24-02

Mauria, R., T01-03

Mayer, A., A06-14, A06-16, B08-04, B08-22, B08-33

Mazor, K.M., A16-05

McAllister, S., T01-11

McAniff, M., A11-17

McArthur, D., A11-06, B08-23, DBB-05

McCabe, J., B01-14

McCarron, R., A11-23, B02-01, B02-02,

B02-07, B02-09, B08-21, B08-30, B17-02

McCarthy, J., A11-13

McCauley, S., A11-13

McColloster, M., A11-14

McCormack, N., B21-02

McCormick, B., B08-32

McCrea, M., B08-15, DBB-04

McCrea, M.A., A11-26, B08-19, T01-04

McCreedy, D., B18-09

McCuddy, W., B08-22

McCullumsmith, R., B20-03

McGuire, J., B20-03

McGuone, D., A05-05, B05-01, B25-02

McInnes, K., A11-02

McKee, A., A11-28

McKinley, T., S02-02

McLean, M., A19-01

McQuiston, B., A06-06

McVicar, A., T01-24

Mcavoy, M., A08-01

Mclauchlin, L., A10-05

Mcmahon, P., A13-05

Meadows, C., A12-04

Meaney, D., A13-01, T01-05

Meaney, D.F., A22-05

Medda, A., A11-10, B08-35

Medina, J., A16-10

Meeker, K., T01-08

Mehalick, M., A11-23, B08-21

Meier, T., A06-14, B08-22, B08-33

Mendelev, N., B02-07

Merchant, J., T01-01

Mercier, C., PL02-01

Meredith-Duliba, T., A13-10, A23-07

Mermagen, W., A05-02

Mertz, M.W., B30-03

Mervis, R., B22-01

Messina, N., B18-02

Metgud, S., B18-02

Metheny, H., B01-10, B11-01

Meyer, B., B08-23, DBB-05

Micci, M., A22-09, B08-12

Micci, M.-A., B23-01, B23-04

Mikhail, M., B27-01

Miller, E., A11-13

Milligan, K., A11-15

Milzman, D., B08-32

Minaeva, O., A11-28, A13-11, B08-06, DBA-05, DBB-03

Mink, R., A13-03, B25-03

Mirshahi, N., A06-14

Misistia, A., B02-08

Misquitta, L., WS06-03

Miyamoto, T., A10-01, A13-08, A21-09
Mocciaro, E., B23-04

Mohaimany-Aponte, A., T01-11

Mohammad, L., A12-04

Moncaster, J., A11-28, B08-06, DBB-03

Moncaster, J.A., A13-11, DBA-05

Mondello, S., A06-08, A06-14, A22-05, A22-07, B08-20, DBA-02, DBA-09

Monson, K., A23-04

Montie, M., A11-16

Moon-Massat, P., B17-02

Moore, A., A16-10, A21-06, A22-04

Moore, C., A03-07, A13-10, A13-16, DBA-01

Moore, J., A11-29

Moore, T., A10-04

Moran, T., A11-29, B08-35

Morel, P., A06-15

Moreno, W., A18-01

Morin, A., B08-24

Moritz, K., A01-08, B21-02

Morley, B., B18-06

Morrison, H., A01-04, B21-03

Morrissey, M., B01-13

Morrissey, M.R., A01-03

Moses, A., B08-13, B08-17

Moskowitz, J., A16-05

Moss, W., B08-06, DBB-03

Mothe, A., A06-04

Motoki, Y., A06-13

Mountney, A., A05-09

Mountz, J., A06-10

Mouzon, B., A08-02, A11-04, A20-01, B01-05, B08-24, DBA-08

Moy, C., B08-07

Moyer, M., A23-07

Mualem, W., B18-14

Mucha, A., A11-18

Muehlschlegel, S., A05-06, A16-05

Mujat, M., A13-11, DBA-05

Mullah, H., B02-01

Mullan, M., A11-04, A20-01, B01-05, DBA-08

Muramatsu, S.-i., B26-02, DBB-09

Murphy, J., B08-32

Muttikkal, T.E., B08-05

Muza, P., A20-01, DBA-08

Najarian, K., A11-32, A11-33, B07-05

Namjoshi, D., A11-02, A11-03

Napier, D., A05-09

Narayan, R., B16-05, B16-06

Nash, M., A22-02

Nassau, K., B01-04

Nassau, K.L., B01-07

Navarro, V., A06-09

Ndode-Ekane, X., B18-01

Nelson, D.W., B17-03

Nelson, L., A11-16, B08-15, DBB-04

Nelson, L.D., A11-26, B08-19, T01-04

Nemoto, E., A21-01

Netoff, T., A09-02

Neustadtl, A., B08-32

Newell, E., T01-20

Newman-Norlund, R., B08-34

Neyra, S., A18-12 
$\mathrm{Ng}, \mathrm{M} ., \mathrm{A} 23-05$

Nguyen, O., A20-03

Nguyen, T., A11-30, B28-05, T01-23

Ngwenya, L., A11-36, A15-02

Nicholas, M., B01-01

Nichols, J., A13-02, B08-25

Niedzielko, T., A22-01, B23-05, B24-03

Nielson, J., AANS02-02, B07-02, B19-01, T01-06

Niemet, P., A21-06, A21-07

Niesman, P.J., B01-07

Nikulina, E., B06-02

Njoku, I., B01-01, B01-08

Noble-Haeusslein, L., B18-09

Noble-Haeusslein, L.J., A22-05

Nojima, J., A06-13

Noor, N., A11-01

Norrell, P., A21-07

Nunez, J., A05-05

O'Brien, M., A11-27

O’Neil, D., B01-08, B01-09

O'Niell, J., A12-04

O'Shea, P., B08-03

Obenaus, A., A13-12, A23-03, A23-05, B25-01

Oddo, S., A01-05

Ogier, M., B18-10

Ogino, Y., A04-01, B08-20

Oglesbee, S., B08-04, B08-33

Oh, B.-M., T01-17

Ohri, S.S., B13-01

Ohtake, Y., B26-02, DBB-09

Ohtori, S., A10-01, A10-03, A13-08, A21-09

Oistilli, C.K., A22-12

Ojo, J., A08-02, A11-04

Ojo, J.O., A20-01, DBA-08

Okada-Rising, S., A18-03, A18-10, DBA-07

Okonkwo, D., A06-10, A11-17, A11-18, A11-34, A11-35, B15-01, B27-02

Okonkwo, D.O., A11-07, A11-08

Okuka, M., B22-01

Olby, N., A13-09

Oleru, A., B08-01

Olsen, A., A13-03, B25-03

Olson, B.B., A13-02

Olson, S., B18-11, B28-04, B28-05, B28-06

Omer, T., T01-25

Ondek, K., B06-04, B06-05

Orantes, A., A23-05

Ordaze, J., T01-23

Orita, S., A10-01, A10-03, A13-08, A21-09

Ornelas, D., A12-06, DBA-04

Osier, N., B15-01

Oster, A., B08-32

Ostrowski, S., B16-03

Ottens, A., A22-08, DBA-10

Oudega, M., A02-02

Owens, J., A20-01, DBA-08

Oxland, T., A07-01, A07-02, A07-06, B11-03

Oyoyo, U., A13-02

Palminha, C.A.P., B18-01

Pandher, M., B18-02
Pandya, B., B09-02

Pandya, C., B09-02

Pandya, J., A05-07, A18-03, A18-10, DBA-07

Panzer, M., A07-03

Panzer, M.B., T01-02

Papadopoulos, Z., A11-19

Paris, D., A08-02, B08-24

Park, B., B28-03

Park, B.H., A12-06, DBA-04

Park, E., A23-01

Park, G., B08-04, B08-33

Park, M., A16-04

Park, P., A11-24

Parker, A., T01-10

Parslet, M., B06-07

Parsley, M., A22-03, A22-09, B06-08, B08-12, B08-18, B23-01, B23-04

Parsley, M.A., A18-04

Parsley, M.O., A18-19, B02-14

Partridge, J., B08-28

Pat, B., A22-01, B24-01, B24-03, T01-11

Patanam, I., A11-27

Patel, A., A11-31

Patel, S., A18-11, B26-01

Paterno, R., B01-10

Pathak, V., A07-09

Patrick, N., A04-03

Patzer, R.K., A11-29

Paxton, H., B08-34

Paydar, A., A13-15, A21-08, T01-12

Payne, R., A04-03

Pearce, W.J., A23-05

Pearson, K., B20-06

Pedersen, R., A18-03, A18-10, DBA-07

Perigo, S., B01-12, T01-16

Perlewitz, M., B25-02

Perrine, S., B08-03

Peter, J., B04-05

Petrov, I., A16-09, A18-04, A22-09, B08-12, B23-04

Petrov, Y., A16-09, A22-09, B08-12, B23-04

Petrov, Y.Y., A18-04

Pevzner, A., B06-05

Pfister, B., B02-05, B02-11, B02-12

Pham, D., A03-07, A09-01, A13-13, A13-14, A13-16, DBA-01

Pham, D.L., A13-10

Phelps, S., B08-35

Phillips, J., A03-06, B08-04, B08-33

Phillips, L., B18-12, DBB-08

Piao, C., B09-03

Pick, C., B02-17, B22-01, B26-03

Pick, C.G., B02-15

Pickens, J., A22-01, B23-05

Pinton, G., A07-04

Pitkänen, A., B10-01, B18-01

Pitkanen, A., A06-09

Pivonka-Jones, J., A13-02

Pizzolato-Heine, K., A05-02

Plesnila, N., B06-03

Pletnikova, O., B22-02

Plyler, R., A22-06, S01-01

Podell, K., B08-34

Podolak, O., A11-11, A11-12
Polk, T., B08-32

Pollock, J., A13-05

Poloyac, S., A18-02, A22-07, DBA-09

Poloyac, S.M., A22-05

Poly, F., B08-21

Poropatich, R., A11-18

Poudel, M., B20-04

Poulsen, D., A06-09

Povlishock, J., A04-01, A22-07, A22-08, B08-20, DBA-09, DBA-10

Povlishock, J.T., A06-08, DBA-02

Powell, M., B18-13

Prabhakara, K., B18-11, B28-04, B28-05, B28-06

Presson, N., A11-18

Price, M., B27-08

Prichep, L., B08-34

Prins, M., B20-01, T01-14

Proctor, J., A16-10

Protzuk, O., A18-13

Prough, D., A06-02, A06-17, A16-09, A22-03, A22-09, B06-07, B06-08, B08-12, B08-18, B19-02, B23-01, B23-04

Prough, D.S., A11-01, A18-04, A18-19, A22-05, B02-14

Prouty, M., B08-21

Puccio, A., A06-10, A11-07, A11-08, A11-17, A11-18, B15-01, B27-02

Puhakka, N., A06-09

Pukenas, B., A22-06

Pultz, D., A11-18

Qaiser, R., A05-08

Qu, W., B27-05

Quinn, C., A14-01

Quinn, D., B26-04

Quinn, T., A16-05

Rabchevsky, A., A18-11, B26-01

Rachmany, L., B02-17

Radabaugh, H., B01-01, B01-04, B01-08, B01-09, B01-11

Radabaugh, H.L., B01-07

Rader, B., B25-05

Radomski, K., A03-02

Rahmathulla, G., A10-05

Ram, O., B02-20, B02-21

RamaRao, K.V., B02-05

Ramer, M., B11-03

Ramos, A., B02-08

Ranganathan, P., A06-11, A06-15, B01-06, B07-03, B07-04, T01-17

Rangghran, P., A16-10

Ranjbaran, M., A11-27

Ranson, J., A11-16, A11-26, B08-19

Rashid, F., A13-03, A23-05, B25-03

Ratcliff, J., A11-29

Ratliff, W., A18-12, B22-01

Ravi, H., A13-14

Ravindra, V., A13-04

Reddy, Y., A23-07

Reed, J.M., A11-04

Reeves, T., B18-12, DBB-08

Regan, R., B16-01 
Regassa, L., B02-10

Reilly, L., B02-08

Repshas, J., A11-14

Rex, T., B02-18

Reynolds, B., B08-05, B08-26

Reynolds, J., B16-02, B24-04, T01-13

Richardson, C., A06-06

Rick, B., B16-04

Riddler, A., A22-08, DBA-10

Righelli, D., A10-02, DBA-03

Riley, P., T01-14

Ritchie, E., B08-27

Ritter, A., A12-02

Rivera, K., A05-03, A05-10, T01-07

Rizk, E., A04-03

Roach, M.J., A10-04

Robel, S., A12-05

Robert, J., A11-03

Robertson, C., A06-02, A06-17, A11-13,

A14-02, A16-07, A16-09, B06-09, B25-04

Robertson, C.S., A22-05

Robinson, C., A01-03, A11-34, A11-35, A11-36

Rockswold, G., A16-03

Rodgers, R., A23-06, S02-02

Rodriguez-Grande, B., B25-01

Rodriguez, E.G., B16-03

Rodriguez, U., B02-14

Rogers, J., A06-06

Rolfe, A., A18-13, B18-13

Roller, S., A21-02, B25-05

Ronna, B., A13-04

Rooks, T., A11-20

Rosen, C., A05-08

Rosen, S., B18-09

Rosenbaum, P., B08-23, DBB-05, T01-16

Rowe, R., A01-04, B08-10, B18-06

Rowe, R.K., A01-05

Rowell, S., B16-04

Rowson, B., A07-03, A11-21

Rowson, S., A07-03, A11-21

Roy, S., A13-13

Ruan, J., A11-30

Rubiano, A., A13-04

Rubin, J., B07-04

Rubio, D., A21-08

Rubovitch, V., B02-15, B02-17, B26-03

Rumbika, K., A20-03

Ruppert, K., B28-04, B28-05, B28-06

Russell, A., B12-01

Russo, R., B11-04, T01-15

Ryan, S., A03-08

Ryman, S., B08-04, B08-33

Ryu, J., A04-02, A11-31, B22-02, T01-25

Saad, N., B18-04, DBB-06

Saatman, K., B08-14, B23-03

Saatman, K.E., A22-05

Saber, M., A01-05

Sabirzhanov, B., A18-16, B21-01, B24-02

Sacktor, T., B06-02

Sadeghpour, S., A04-02

Sadot, O., B02-20, B02-21

Saha, B., B17-02
Sahyouni, R., A15-01

Saigal, R., AANS02-02, T01-06

Saito, J., A10-01, A10-03, A13-08, A21-09

Sajja, S., B02-03

Salehi, A., A23-03

Samadani, U., A06-06, A09-02

Sanchez-Molano, J., A18-01

Sanchez, C.A., A07-02

Sanchez, E., A07-03

Sanchez, I., B08-31

Sanders, B., A07-05

Sands, M., A22-12

Sandsmark, D., A13-10, A23-07

Sangobowale, M., A23-07

Sanjakdar, S., A05-09

Santhakumar, V., B02-16

Saraswati, M., B06-09, B25-04

Sargolzaei, S., A06-12, A13-15, T01-16

Sarkar, C., B04-03, B04-04, B04-05, DBB-02

Satris, G., B01-13

Sauerbeck, A., B29-03

Savarraj, J., A22-04

Sayed, N., A11-14

Scafidi, S., B20-04

Scalea, T.M., A05-06

Schafer, M., B13-02

Scheidt, M., A11-27

Schmidt, C.E., B30-03

Schneider, B., B08-03

Schneider, L., B14-01

Schneider, W., A06-10, A11-07, A11-08, A11-17, A11-18

Schnettgoecke, B., A01-06

Schnyer, D., B08-34

Schreiber, S., B02-15

Schurman (nee O’Brien), L., B18-12, DBB-08

Schuster, A., T01-17

Scott, D., B23-03

Scott, G., A03-04

Scott, T., B01-15

Scultetus, A., B17-02

Seabury, S., B08-09

Seira, O., T01-18

Sell, S., A22-09, B08-18

Seth-Hunter, D., A11-27

Shacham, S., B03-01

Shaff, N., B08-33

Shah, A.S., B08-19

Shah, N., B21-03

Shahlaie, K., B06-05

Shandra, O., A12-05

Shapiro, M.S., B08-31

Sharkey, L., A07-02

Sharma, S., A01-07, A11-34, A11-35, A11-36

Sharpless, J., A11-07, A11-08, A11-18

Shaver, T., B01-15

Shaw, G., A06-14

Shea-Donohue, T., B27-06

Shear, D., A05-01, A05-03, A05-04, A05-07, A05-09, A05-10, A06-03, A06-08, A12-01, A12-03, A18-03, A18-10, A22-07, DBA-02, DBA-07, DBA-09
Shear, D.A., A22-05

Shekhar, A., B27-05

Shen, J., B25-02

Shen, S., A06-16

Sherman, S., A03-06

Sheth, K.N., A05-06

Shibuya, M., B04-02

Shields, C.B., T01-23

Shimada, R., B04-02

Shu, S., B25-04

Shultz, S.R., A22-05

Shupe, E., B12-01

Shutter, L., A16-05

Shuttleworth, C., A21-01

Siddiqui, A., B28-01

Sieg, E., A04-03

Silbergleit, R., A16-03

Sillanpää, M., B10-01

Silverman, E., A03-07, A13-10, A13-16, DBA-01

Sindelar, B., A11-22

Singh, C., A21-03, B13-02

Singh, I., A18-06, A18-07, A18-09

Singh, S., B08-23, DBB-05

Siverman, E., A23-07

Skotak, M., B02-05, B02-13, B02-16

Sloley, S., B08-28, T01-09

Smith, A., A21-02, A21-07, B21-04, B25-05, B27-06

Smith, C., B28-02

Smith, D., A03-03, A03-05, B03-03, B11-04, DBB-01, T01-10, WS06-03

Smith, D.H., A03-08, A17-01, A22-05

Smith, T., B01-05, B18-12, DBB-08

Smith, W., A16-06

Soldozy, S., B08-26

Soltanian-Zadeh, H., B08-16

Song, S.-K., B22-03

Soose, R., A11-18

Soroushmeh, S.M.R., B07-05

Soroushmehr, S.M.R., A11-32, A11-33

Sowers, J., B19-02

Speidel, J., A07-06, B11-03

Sprague, S.M., B08-31

Spratt, H., A06-02

Spratt, H.M., B19-01

Springer, J.E., B27-07

Spry, M., B20-06, B27-07

Spurlock, M., A05-03, A05-10, T01-07

Srinivas, S., A21-08

Srinivasan, A., A11-33

Srivastava, A., B28-05

Stabenfeldt, S., A22-10

Stanton, A., B08-05, B08-26

Stanton, P., A11-28

Statz, J., A11-23, B08-30

Stefos, K., T01-09

Stein, D.G., B17-05

Stein, D.M., A05-06

Steinmetz, M., A10-04

Stemper, B.D., B08-19

Stephen, B.-A., A06-03

Stewart, W., A17-01, B03-03, DBB-01, WS05-01

Stippler, M., A08-01 
Stoddard, E., A15-02

Stoica, B., A18-16, B18-03, B21-01, B27-06

Stolow, M., A22-06

Storey, E., A11-11, A11-12, B08-33

Stowlow, M., S01-01

Strain-Malamakal, M., B24-04

Strain, M., B16-02, T01-13

Stukas, S., A11-02, A11-03

Suehiro, E., A06-13

Suen, C., A11-36

Suen, G., B25-05

Sukumar, G., B21-02

Sullivan, P., A18-11, B08-14, B20-06

Sullivan, P.G., B27-07

Sun, D., A18-13, B18-13

Sun, J., A03-10, A18-03, A18-10, DBA-07

Sun, P., B22-03

Sun, W., A13-12

Sun, Y., B27-05

Susick, L., B08-03

Suzuki, M., A06-13

Svensson, M., B17-03

Swallow, J., A11-24

Swanson, R., T01-10

Swietek, B., B02-16

Symes, A., A11-19

Szabo, C., B23-04

Szaflarski, J., A22-01

Szerlip, N., A11-24

Szigeti-Buck, K., B04-01

Szu, J., A12-06, DBA-04

Tabackman, A., B08-17

Tagge, C., B08-06, DBB-03

Tai, W., B26-05, DBB-10

Tajiri, N., B03-01

Tamir, S., B03-01

Tamrakar, P., A16-10

Tang, J., A23-05

Tang, M., A18-12

Tarapore, P., A05-11, A11-36

Tarumi, T., A11-14

Tator, C., A06-04

Tavanaiepour, D., A10-05

Taylor-Burds, C., A07-07

Taylor, B., B08-34

Taylor, S., A01-03

Tcherepanov, A., B06-02

Techar, K., A06-06

Temkin, N., B01-13

Teng, Y., A20-03, A22-11

Tenlep, S.N., B20-06

Tenovuo, O., B10-01

Terasaki, Y., S01-03

Terminel, M., B04-06

Terpolilli, N.A., B06-03

Terry, B., A03-04

Teter, O., A13-01

Tetzlaff, W., A07-01, A07-06, B11-03, T01-18

Thelin, E., B17-03

Thibeault, C., A11-27

Thiels, E., B01-06

Thomas, T., B21-03
Thomas, T.C., B08-02

Thompson, H., B24-05

Thompson, P., A13-03, B25-03

Thomsen, G., A11-25

Thorpe, M., A06-06

Thorpe, S., A11-27

Timmons, S., A04-03

Timothy, K., A23-06

Titus, D., B06-01, B06-06

Tobias, E., A15-01

Todd, B., T01-20

Tomasek, J., B16-03

Tong, K., A13-02

Torres, I., A18-17

Tortella, F., A06-08, A12-03, A22-07, DBA-02, DBA-09

Toussia-Cohen, S., B26-03

Tran, D.K.T., A15-01

Travis, C.G., A18-03

Treble-Barna, A., A12-02

Treu, R., A18-01

Troncoso, J., B22-02

Truettner, J., A18-01

Tsai, E., B28-02

Tschiffely, A., A11-23, B08-30

Tsibris, J., B22-01

Tu, T.-W., B20-05

Tucker, L., B01-14

Tummala, S., T01-05

Tupper, D., A06-06

Turner, J., T01-03

Turner, M., A11-14

Turner, O., A22-01

Turtle, J., T01-13

Turtzo, C., B08-13

Turtzo, L.C., B08-17, B08-29

Tweedie, D., B02-17

Ubel, P., A16-08

Uche, V., B08-17

Ullman, J., A01-02

Ulndreaj, A., T01-22

Ulyanova, A., B11-04

Umali, M., B02-07

Unger, E., B17-02

Untereiner, A., B23-04

Upadhyayula, P., A11-36

Urankar, S., B23-02

Uray, K., A23-02

Urioste, R., B02-03

Uy, K., A14-01

Vaibhav, K., B09-02, B18-04, B18-05, DBB-06

Valadka, A., A11-34, A11-35, B03-02, B27-03

Valadka, A.B., A22-05

Vallabhaneni, P., A11-04

Van Meter, T., A06-14

van der Merwe, A., B08-29

VanAlbert, S., B02-03

VandeVord, P., A02-03, A22-05, B02-04, B02-06

Vascak, M., A03-10
Vaughan, L., B07-04

Vedantam, A., A14-02, A16-07

Vedung, F., T01-21

Vekaria, H., B08-14, B20-06

Vekariab, H., B27-07

Veksler, R., A06-14

Veldhorst, A., T01-24

Velosky, A., B01-14

Veltri, L., B01-15

Verala, L., B04-01

Veraza, R.J., B08-31

Verma, R., T01-10

Vespa, P., A13-06, A13-07

Vest, V., B02-18

Vickers, J., A01-04

Vidal, P., T01-22

Vigil, F., B08-31

Vijapur, S., A06-15

Villalon-Reina, J., A13-03, B25-03

Villapol, S., B18-03, B18-14, T01-09

Vincent, P., A04-02

Viollet, C., B21-02

Viox, J., B22-03

Vlasova, R., B25-03

Von Leden, R., A01-08

Vuokila, N., A06-09

Wachs, R.A., B30-03

Wade, C., A01-06, B16-03

Wagner, A., A06-11, A06-15, A12-02, B01-06, B07-03, B07-04, T01-17

Wagner, K., A01-02

Wahlin, R.R., B17-03

Wainwright, M., B09-03

Walilko, T., B02-06

Walker, K., B28-02

Walker, P., A11-23, B08-30

Walker, S., B08-20

Wall, A., T01-21

Wallendorf, M., B22-03

Walsworth, M., A23-03

Wang, D., B08-32

Wang, E., B08-32

Wang, J., A18-06, A18-07, A18-09, B18-08, DBB-07

Wang, K., A06-01, A06-08, A06-15, A18-17, A22-07, B08-20, DBA-02, DBA-09

Wang, L., A11-11, A20-03

Wang, L.-L., B26-05, DBB-10

Wang, W.-T., A13-14

Wang, W.-X., B27-07

Wang, X., A20-04, S01-03

Wang, Y., B02-03, B27-05

Wang, Z., B07-03, B08-23, DBB-05

Wanner, I., A06-14, A06-16

Ward, K., A11-32, A11-33, B07-05

Ware, A., B08-34

Warren, E., A06-06

Warth, A., B20-01

Wassarman, D., S02-03

Wasserman, E., A03-07, A13-10, A13-16, DBA-01

Wasserott, B., B08-04, B08-33

Webb, A., B08-32 
Weber, M., A03-03, B03-03, DBB-01, T01-10, T01-15

Webster, M., A22-02

Weisz, H., A06-02, A06-17

Wellcome, J., B01-04

Wellington, C., A11-02, A11-03

Welsbie, D., A11-31

Wertz, C., B08-04, B08-33

Whalen, M., B04-07

White, A., B17-04

White, F., S02-02

Whiteman, A., A12-01

Whitney, K., B06-02

Whyte, T., A11-02

Wicker, E., B08-28, B18-14

Wiebe, D., A11-12

Wiggins, J., B26-06

Wild, A., A11-26

Wilde, E., A11-13, B08-34

Wilder, D., B02-03

Wilfred, B.S., B16-07

Wilk, S., A11-27

Wilkinson, A., A11-02, A11-03

Williams, D., A11-18

Williams, E., B24-05

Williamson, C., A11-32, A11-33, B07-05

Williamson, T., A16-08

Willsey, K., A11-24

Wilson, G., B26-06

Wilson, N., A18-14, B06-01, B06-06

Wilson, V.A., A18-15

Winkler, E., A05-11, A11-34, A11-35, A11-36

Winkler, M., B11-02

Winston, C., B08-28

Wintermark, M., B08-26

Wiseman, N., B08-16

Witcher, K., B18-07

Witko, J., B20-05

Witten, A., A22-10

Woitzik, J., B11-02

Wojnarowicz, M., A11-28, B08-06, DBB-03

Wojnarowicz, M.W., A13-11, DBA-05

Wolf, J., B06-07, B11-04, B19-01, T01-15

Wolfgang, M., B20-04

Wood, B.D., B13-01

Wright, D., A11-10, A11-29, B08-35
Wu, D., A11-29

Wu, J., A18-16, B04-03, B04-04, B12-01, B24-02, DBB-02

Wu, J.-Y., B08-28

Wu, L., A11-30, B04-07, B08-36

Wu, P., A11-01, B19-02

Wu, S., A16-02, DBA-06

Wu, T., B08-34

Wu, W., A18-15, T01-23

Wu, X., A18-15, B27-05

Wu, Y., A03-06

Wu, Z., A18-18

Xia, Y., B27-05

Xing, C., S01-03

Xiong, G., B01-10

$\mathrm{Xu}, \mathrm{L} ., \mathrm{A} 04-02$, A11-31, T01-25

$\mathrm{Xu}, \mathrm{X} ., \mathrm{A} 20-04$

Xu, X.-M., A18-15, B27-05, T01-23

Yadikar, H., A18-17

Yamamoto, S., A16-09, B06-08

Yamazaki, M., A10-01, A10-03, A13-08, A21-09

Yan, H., A18-02

Yan, L., B02-16

Yang, K., A18-08

Yang, Q., A18-18

Yang, W., A12-01

Yang, X., A05-04, A05-07, B16-07

Yang, Y., A21-01

Yang, Z., A06-15

Yao, H., A11-32, A11-33, B07-05

Yap, P.-T., A13-09

Yarmush, M., A21-04

Yarnell, A., B02-07

Yaszemski, A., B21-05

Yauger, Y., B21-02, B21-05

Ye, Z., B22-03

Yeargin, S., B08-34

Yeary, J., B25-06

Yeates, K., B08-04

Yeo, R., B08-04, B08-33

Yin, L., B18-04, DBB-06

Yonas, H., A12-04

Yoon, Y., A18-08

Yost, R., A18-17
You, Y., A20-04

Young, J., B27-08

Young, W., B18-02

Younger, D., B02-05, B02-13

Yousuf, S., B17-05

$\mathrm{Yu}, \mathrm{A}$., A07-08

Yu, C.-G., A22-12

$\mathrm{Yu}, \mathrm{J} ., \mathrm{B} 24-02$

Yu, L., A18-16

Yu, X., A03-01

Yue, J., A01-03, A01-07, A05-11, A11-34, A11-35, A11-36

Yvette, C., A12-02

Zac, C., B02-07

Zack, D., A11-31

Zafonte, R., A22-11, B07-02

Zahid, A.B., A06-06

Zai, L., B02-06

Zambrano, R., A16-02, DBA-06

Zane, F., A23-06

Zeng, X., A22-11

Zeng, Y., A18-19

Zhan, J., B22-03

Zhang, B., T01-24

Zhang, C.-L., B26-05, DBB-10

Zhang, J.H., A23-05

Zhang, K., B19-02

Zhang, L., A07-09, A22-05, B02-19

Zhang, R., A11-14

Zhang, S., A18-13, B18-13

Zhang, X., B27-02

Zhang, Y., B04-01, T01-23

Zhang, Z., B06-09

Zhao, S., B08-35

Zhao, W., A11-05, B08-36

Zhou, R., A07-09

Zhu, B., B01-15

Zhuo, J., B08-16

Ziebell, J., A01-04

Zilberstein, Y., B02-15

Zimmermann, L., AANS02-02, T01-06

Ziogas, N., A05-02, A11-31, B22-02, T01-25

Zuckerman, A., B02-20, B02-21

Zurhellen, C., B17-06

Zusman, B., A11-07, A11-08 